\title{
Cross-Regulation of Innate and Adaptive Immunity: A New Perspective for the Pathogenesis of Inflammatory Bowel Disease
}

\author{
Da Yeon Oh and Seong-Joon Koh \\ Department of Internal Medicine, Seoul National University Boramae Hospital, Seoul National University College of Medicine, Seoul, Korea
}

See "Expression of TIM-3, Human $\beta$-defensin-2, and FOXP3 and Correlation with Disease Activity in Pediatric Crohn's Disease with Infliximab Therapy" by Mi Jin Kim, et al. on page 370, Vol. 9. No. 3, 2015

Inflammatory bowel disease (IBD) is characterized by chronic, relapsing inflammation, resulting from a pathological response in the gastrointestinal tract. ${ }^{1}$ The pathogenesis of IBD remains obscure; however, it has been suggested that IBD is caused in genetically susceptible host by an appropriate immune response against abnormal intestinal microflora. ${ }^{2}$ Abnormal innate and adaptive immune responses are regarded as the major components of IBD pathogenesis. For several decades, a majority of studies related to IBD have focused on the abnormal adaptive immune response as Crohn's disease (CD) is considered to be associated with a Th1 response, while ulcerative colitis is driven by a Th2 response. ${ }^{3}$ Recent advances in IBD research, such as data from a genome-wide association study and the results of microbiome analyses, have helped unravel the complex innate immune responses in IBD, such as mucosal barrier function, microbial sensing, autophagy, and endoplasmic reticulum stress; these have indicated the importance of cross regulation of the innate and adaptive immune responses.

The innate immune system, which provides nonspecific and rapid response, is at the head of host defense against intestinal pathogens. The innate immune system is composed of multiple factors such as antimicrobial peptides (such as $\beta$-defensin-2), and performs the diverse functions, including epithelial barrier function, autophagy, and unfolded protein response. ${ }^{4-6}$ In addition, this systems consists of phagocytic cells, such as dendritic cells, macrophages, and intestinal epithelial cells, which can recognize the intestinal microbial antigens, causing a rapid inflammatory response against abnormal pathogens. ${ }^{7}$ Furthermore, antigen presenting cells, such as dendritic cells, secrete cytokines that facilitate $\mathrm{T}$ cell differentiation, resulting in the activation of the adaptive immune systems. Unlike the innate immune system, the adaptive immune system is highly specific and confers long-lasting immunity. T cells are key players in the adaptive immune system. Naive T cells can differentiate into various effector T cells, such as Th1, Th2, or Th17 cells. These T cell subsets contribute to the removal of microbial organisms, such as bacteria, fungi, and parasites. ${ }^{8}$ However, the dysregulation of activated $\mathrm{T}$ cells results in the secretion of proinflammatory cytokines and chemokines, which leads to the onset of the chronic inflammation that characterizes IBD.

The interplay between the innate and adaptive immune systems plays a central role in the pathogenesis of IBD. However, research into the pathogenesis of IBD has been largely focused on the two immune systems separately, some have focused on the adaptive immune system, especially $\mathrm{T}$ cells, whereas others have investigated the intestinal epithelial cells or phagocytic cells expressed in the lamina propria. The recent advances in molecular biology techniques, including sequencing, have aided in exploring the cross-talk between the innate and adaptive immune responses. For example, NOD2, a pattern recognition receptor belonging to the innate immune system, play a role in the regulation of adaptive immune responses. ${ }^{9}$ Despite this, the cross-regulation of the innate and adaptive immune systems remains largely unexplored.

An article published in the latest issue of Gut and Liver has demonstrated the close association between the innate and adaptive immune systems. ${ }^{10}$ In this study, the authors investigated the frequency of expression of $\mathrm{T}$ cell immunoglobulinand mucin-domain-containing molecule-3 (TIM-3), human $\beta$-defensin-2 (HBD-2), forkhead box protein-3 (FOXP-3) and

Correspondence to: Seong-Joon Koh

Division of Gastroenterology \& Hepatology, Department of Internal Medicine, SMG-SNU Boramae Medical Center, Seoul National University College of Medicine, 20 Boramae-ro 5-gil, Dongjak-gu, Seoul 156-707, Korea

Tel: +82-2-870-2234, Fax: +82-2-870-3866, E-mail: jel1206@snu.ac.kr pISSN 1976-2283 eISSN 2005-1212 http://dx.doi.org/10.5009/gnl15123

@) This is an Open Access article distributed under the terms of the Creative Commons Attribution Non-Commercial License (http://creativecommons.org/licenses/by-nc/3.0) which permits unrestricted non-commercial use, distribution, and reproduction in any medium, provided the original work is properly cited. 
$\mathrm{CD}^{+} / \mathrm{CD} 25^{+} / \mathrm{FOXP}^{+}$regulatory $\mathrm{T}$ cells (Tregs) and their correlation with the clinical activity in pediatric patients with $\mathrm{CD}$, subjected to infliximab therapy for a year. TIM-3 is a specific marker for Th1 cells, which is known to negatively regulate the $\mathrm{T}$ cell response. The expression of TIM-3 mRNA was higher in the colonic mucosa prior to infliximab therapy compared to heathy controls. In contrast, TIM-3 mRNA expression was lower in the peripheral blood mononuclear cells of patients with $\mathrm{CD}$, compared to the healthy controls. The TIM-3 mRNA expression increased in the peripheral blood mononuclear cells of CD patients treated with infliximab; however, corresponding decrease was observed in the colonic mucosa. The expression of HBD-2, an antimicrobial protein, increased in response to the invasion of pathogens or enteric microbiota. The expression of $H B D-2$ mRNA was observed to be higher in the colonic mucosa of $\mathrm{CD}$ patients compared to healthy controls. The enhanced HBD-2 expression was suppressed after infliximab therapy. The expression of FOXP-3, a transcription factor for regulating the differentiation of Treg cells, was also increased in the colonic mucosa of active CD patients (and subsequently decreased after infliximab therapy). In addition, there was an increase in the proportion of $\mathrm{CD}^{+} / \mathrm{CD} 25^{+} / \mathrm{T}$ cells in $\mathrm{CD}$ patients after infliximab therapy.

Taken together, this study suggested an association between the innate and adaptive mucosal immune systems in CD pathogenesis. Therefore, these results could indicate the crossregulation of the innate and adaptive immune systems in IBD. However, several critical issues need to be resolved. First, the isolation of specific cells using a sorting system may provide precise data regarding the cross-regulation of the two systems. Second, a large number of patients, including adult patients with IBD, must be analyzed in order to confirm the results and elucidate the mechanism of cross immune regulation. Finally, the molecular basis of cross regulation must be elucidated.

In summary, innate and adaptive immune systems are critical for the induction of chronic inflammation in IBD. Despite this, the intestinal mucosal innate and adaptive immune systems have been studied separately for a long time. Research into either system alone has not aided in the complete understandings of the pathogenesis of IBD. Future studies must focus on the cross-regulation of the mucosal innate and adaptive immune systems; in addition, both immune systems must be subjected to joint investigations. This would facilitate the identification of new therapeutic targets for IBD and the understanding of the molecular basis of these targets.

\section{CONFLICTS OF INTEREST}

No potential conflict of interest relevant to this article was reported.

\section{REFERENCES}

1. Baumgart DC, Sandborn WJ. Inflammatory bowel disease: clinical aspects and established and evolving therapies. Lancet 2007;369: 1641-1657.

2. Han DS. Current status and prospects of intestinal microbiome studies. Intest Res 2014;12:178-183.

3. Fuss IJ, Neurath M, Boirivant M, et al. Disparate CD4+ lamina propria (LP) lymphokine secretion profiles in inflammatory bowel disease. Crohn's disease LP cells manifest increased secretion of IFN-gamma, whereas ulcerative colitis LP cells manifest increased secretion of IL-5. J Immunol 1996;157:1261-1270.

4. Geremia A, Biancheri P, Allan P, Corazza GR, Di Sabatino A. Innate and adaptive immunity in inflammatory bowel disease. Autoimmun Rev 2014;13:3-10.

5. Buisine MP, Desreumaux P, Debailleul V, et al. Abnormalities in mucin gene expression in Crohn's disease. Inflamm Bowel Dis 1999;5:24-32.

6. Kim JM. Antimicrobial proteins in intestine and inflammatory bowel diseases. Intest Res 2014;12:20-33.

7. MacDonald TT, Monteleone I, Fantini MC, Monteleone G. Regulation of homeostasis and inflammation in the intestine. Gastroenterology 2011;140:1768-1775.

8. Romagnani S. Lymphokine production by human $\mathrm{T}$ cells in disease states. Annu Rev Immunol 1994;12:227-257.

9. Siegmund B, Zeitz M. Innate and adaptive immunity in inflammatory bowel disease. World J Gastroenterol 2011;17:3178-3183.

10. Kim MJ, Lee WY, Choe YH. Expression of TIM-3, human $\beta$-defensin-2, and FOXP3 and correlation with disease activity in pediatric Crohn's disease with infliximab therapy. Gut Liver 2015;9:370-380. 